\title{
Panhysterectomies-A Comparison of Perioperative Care of Abdominal and Laparoscopic
}

\author{
Chunhua Zhang ${ }^{1}$, Silan Ren ${ }^{2}$ \\ ${ }^{1}$ Department of Gynecology, First Xiangya Hospital of Central South University, \\ Changsha, Hunan 410008, P.R. China; \\ ${ }^{2}$ Department of Nursing, Sichuan Vocational College of Health and \\ Rehabilitation, Zigong, Sichuan 643000, P.R. China.
}

First Author: Chunhua Zhang, Nursing Supervisor Department of Gynecology, First Xiangya Hospital of Central South University, Changsha, Hunan 410008, P.R. China.

Corresponding Author: Chunhua Zhang. Email: 573700834@ qq.com.

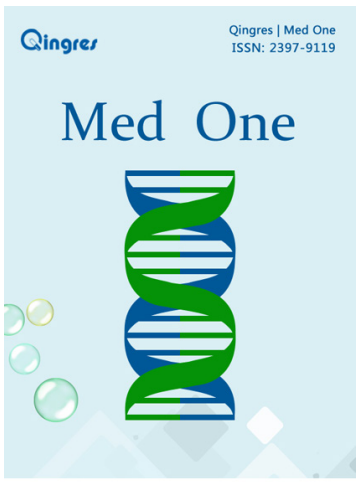

http://mo.qingres.com

\section{GOPEN ACCESS}

DOI: $10.20900 / \mathrm{mo} .20160018$

Received: May 21, 2016

Accepted: July 15, 2016

Published: August 25, 2016

Copyright: ๑2016 Cain et al. This is an open access article distributed under the terms of the Creative Commons Attribution License, which permits unrestricted use, distribution, and reproduction in any medium, provided the original author and source are credited.

\begin{abstract}
Background: To explore the curative effects of laparosopic panhysterectomies and summarize perioperative care experience.

Methods: Forty cases of perioperative hysterectomy, twenty laparoscopic hysterectomies and twenty abdominal hysterectomies were selected for a controlled analysis of treatment efficacies of different nursing measures.
\end{abstract}

Results: Laparoscope surgery durations were longer than abdominal surgery durations. Laparoscopic surgery had lesser bleeding, quicker postoperative ambulation times, shorter intestinal bowel function recovery times, lower patient stress, better patient quality of life, shorter hospitalization stays, and reduced patient economic burdens. Laparoscopic surgery resulted in fewer postoperative complications, reduced nursing care, which improved care effectiveness, and significantly increased patient satisfaction with nursing care.

Conclusion: Laparoscopic panhysterectomies have advantages, including smaller abdominal incisions, shorter hospital stays, improved bed turnover rates, low infection rates, and fast recovery. Its curative effect is superior to the abdominal hysterectomy and it is more suitable clinically. Increased patient supervision and care given during the perioperative period facilitates post-operative healing. 
Key Words: Laparoscope; Panhysterectomy; Perioperative care

\section{INTRODUCTION}

The panhysterectomy is usually used to heal endometrial lesions, hysteromyoma, adenomyosis and other gynecopathy symptoms. The traditional panhysterectomy mostly focuses on the abdominal hysterectomy and hysterectomy via the vagina. During the increasing perfection process, laparoscopic surgery has been widely applied due to its own advantages, including small bleeding amount, fast healing and recovery, improved quality of life of patients, low infection rate, fewer complications and shorter hospital stay. The clinical data of 80 patients with the laparoscopic panhysterectomy from July 2013 to June 2014 were compared and analyzed in this study.

\section{SUBJECTS AND METHODS}

\subsection{Subjects}

Eighty patients were equally divided into two groups at random: abdominal group and laparoscopic group. Abdominal group was the control group, aged $37-70$ years, with a mean age of 50.1 years old. It was composed of 40 patients, including 19 patients with hysteromyoma, 9 patients with adenomyosis, 1 patient with cervical intraepithelial neoplasias level III, and 11 patients with endometrial lesions; 9 patients were concurrently with hypertension and 10 patients concurrently with diabetes. Laparoscopic group was the experimental group, aged $38-69$ years old, with a mean age of 49.5 years, including 18 patients with hysteromyoma, 10 patients with adenomyosis, 2 patient with cervical intraepithelial neoplasias level III, and 10 patients with endometrial lesions, of which, 11 patients were concurrently with hypertension and 6 patients concurrently with diabetes. No statistical significance existed for age, disease condition, education level, and other dimensions in the patients of two groups $(p<0.05)$, namely, they were experimentally comparable. All patients had no contraindication against anesthesia and surgery.

\subsection{Methods}

The following cares were taken during the surgery of the laparoscopic group: 1) Do not insert the catheter and actively encourage and guide patients for an early ambulation to make stool visit at bedside or in toilet after the surgery; 2) Encourage patients for an early ambulation or to make the physical therapy at the site of pain to alleviate the pain of some patients with shoulder-backache after the surgery, under the condition without patients controlled analgesia (PCA), thus helping the patients recover gastrointestinal functions as early as possible; 3 ) Use the new scientific methods as per the dietary requirements before the surgery: light digestible and semi-liquid food were eaten, and no food or drinking was eaten since 22:00 pm the day before surgery; 4) Reduce the accompanying pressure after the surgery; 5 ) Backache symptoms may appear for the patients subjected to laparoscopic surgery, and no backache may appear for the patients used catheter and PCA in uterine abdominal group. The nurses should explore better ways to minimize the complications in laparoscopic group; and 6) The operation time of laparoscopic group and abdominal group was compared.

\subsection{Perioperative Care}

The patients in abdominal group were nursed as per the usual methods and the patients in laparoscopic group were for the perioperative care.

\subsubsection{Preoperative Care}

\subsubsection{Psychological Care}

The related responsible nurses should pay patrol visit to the surgical patient $24 \mathrm{~h}$ prior to the surgery according to the surgery requisition to master the basic information and disease conditions of the patient, including weight, body temperature, blood pressure, and the like. They should allow the patient and its families to understand the relevant issues and attentions, surgical procedure, and advantages in the surgery process, eliminate the patient's anxiety and fear mentality, and let the patient to understand and be confident in the surgery. The relevant studies showed that the psychological anxiety of the patients before the surgery would significantly affect the surgery effect and the postoperative recovery ${ }^{[1]}$. The practical survey results suggest that the patients had certain psychological anxiety and fear and their average Hamilton Anxiety Scale scores had exceeded 13 points.

\subsubsection{Routine Examinations}

The routine examinations include liver, kidney functions, blood type, chest x-ray examination, blood 
routine examination, bleeding and blood coagulation time, electrocardiogram, etc. Red cell suspension (RCS) of $200 \mathrm{ml}$ was prepared $24 \mathrm{~h}$ before the surgery. Pulse, blood pressure, body temperature, breathing and other indicators should be checked prior to the surgery.

\subsubsection{Skin Preparation}

The patient was urged to wash hair, cut nails, shower and change clothes, and other personal hygiene 24 $\mathrm{h}$ prior to the surgery. The jewelry and dentures were not carried into the operating room. The wrist band was improved, including name, bed number, gender, and inpatient number of the patient. The pubic hairs and the skin were shaved. Skin preparation was from xiphoid to midaxillary line to the position of $1 / 3$ thigh (including the vulva). The puncture site for laparoscopic surgery was at the navel, so the navel umbilication was completely cleaned. Specific steps were as follows: scrub the navel umbilication with turpentine and $75 \%$ alcohol before and after the surgery to avoid the wound infection after the surgery.

\subsubsection{Preparation of Vagina}

The surgery of patients could not be performed in their menstrual period. Anti-inflammatory hemostasia measures should be taken for the patients with constant and endless bleeding. The uterus should be cleaned if it was required. The vagina should be prepared and lavaged by diluents - complex iodine - within 3 days before the surgery, with a lavage frequency of twice a day, especially at least once in the morning of the surgery day, and the aim is to clear the ascend bacterial infection distributed in the vagina and avoid the influence on postoperative recovery.

\subsubsection{Gastrointestinal Preparation}

Laparoscopic patients ate a largely fiber-free, digestible semi-liquid food the day before surgery. Abdominal patients could not eat food capable of generating intestinal gas such as high-carbohydrate foods, milk or soy milk to avoid gastrointestinal flatulence. No food or fluid occurred after 22:00 pm the night before surgery. Patients took sulfate-free polyethylene glycol eletrolyte powder for catharsis during the afternoon before surgery. Lavation was made once the night before, or the morning of, surgery in order to empty the gastrointestinal and facilitate the exposure of the operative field and reduce the likelihood of post-operative complications.

\subsubsection{Post-operative Care}

Patients were returned to the ward and their families informed of surgical information and related matters. They were transferred to the ward nurses. Oxygen was administered to comatose patients at the rate of $2-3 \mathrm{~L} /$ rain. Patients rested on their backs without a pillow for $6 \mathrm{~h}$, with their head toward to one side ${ }^{[2]}$. Patients were encouraged to ambulate 6-24 h after the surgery to reduce bloating. In general anesthesia cases, eating was available upon regaining consciousness. In most cases, patients could drink water, and eat a bland nutrient soup $6 \mathrm{~h}$ after surgery. Nurses followed up and observed BP, R and $P$ changes in real-time manner for 24 hours after surgery, and closely monitored patient complexion and attitudes, observed the wound dressing, and whether there was vaginal bleeding. During the perioperative period, the patients were encouraged to urinate.

\section{RESULTS}

\subsection{Operation Effect}

All patients rehabilitated and were discharged after surgery. Abdominal group rehabilitation times, for routine surgery, were longer than the laparoscopic group. The amount of surgical bleeding, catheter pull-out time, period to ambulation, gastrointestinal function recovery period, hospitalization period, and other indicators for the laparoscpic group were significantly shorter than the abdominal group. The specific parameters appear in Table 1.

Table 1. Statistical Surgery Comparison of Different Groups ( $\pm s$ )

\begin{tabular}{lccccccc}
\hline Group & Cases $\begin{array}{c}\text { Surgery } \\
\text { (min) }\end{array}$ & $\begin{array}{l}\text { Bleeding Pull-out } \\
\text { Amount } \\
\text { (ml) }\end{array}$ & $\begin{array}{l}\text { Duration } \\
\text { (h) }\end{array}$ & $\begin{array}{l}\text { Pre-ambulation } \\
\text { Duration(h) }\end{array}$ & $\begin{array}{l}\text { Gastrointestinal } \\
\text { Function Recovery } \\
\text { Period (h) }\end{array}$ & $\begin{array}{l}\text { Hospitalization } \\
\text { Duration (day) }\end{array}$ \\
\hline $\begin{array}{l}\text { Laparoscopic } \\
\text { Group }\end{array}$ & 40 & $110 \pm 25$ & $45-85$ & 0 & $25 \pm 3.1$ & $25 \pm 3$ & $6 \pm 2.3$ \\
$\begin{array}{l}\text { Abdominal } \\
\text { Group }\end{array}$ & 40 & $80 \pm 40$ & $95-145$ & $47 \pm 4.2$ & $48 \pm 3.2$ & $32 \pm 7$ & $11 \pm 2.4$ \\
$p$ Value & $<0.02$ & $<0.02$ & $<0.01$ & $<0.02$ & $<0.04$ & $<0.01$ \\
\hline
\end{tabular}


Patients were returned to the ward and their families informed of surgical information and related matters. They were transferred to the ward nurses. Oxygen was administered to comatose patients at the rate of $2-3 \mathrm{~L} /$ rain. Patients rested on their backs without a pillow for $6 \mathrm{~h}$, with their head toward to one side ${ }^{[2]}$. Patients were encouraged to ambulate 6-24 $\mathrm{h}$ after the surgery to reduce bloating. In general anesthesia cases, eating was available upon regaining consciousness. In most cases, patients could drink water, and eat a bland nutrient soup 6 h after surgery. Nurses followed up and observed $B P, R$ and $P$ changes in real-time manner for 24 hours after surgery, and closely monitored patient complexion and attitudes, observed the wound dressing, and whether there was vaginal bleeding. During the perioperative period, the patients were encouraged to urinate.

\subsection{Complications}

The abdominal group, had: 2 LEDVT patients, 4 instances of bloating, 1 with a wound infection, and 1 with urinary retention. The laparoscopic group had: 2 bloating and shoulder-back pain patients. The probability of complications in the abdominal group was $19 \%$ and $5 \%$ in laparoscopic group with a significant difference $(p<0.05)$.

\subsection{Patients' Satisfaction Comparison between Two Groups}

Patient satisfaction was $99 \%$ with the laparoscopic group and $78 \%$ with the abdominal group and statistically significant $(p<0.05)$. Surgical success includes patient providing effective psychological care which allows them to establish confidence in the treatment and cooperate with staff ${ }^{[3]}$. Pre-operative routine examinations ensure surgical safety, while the preoperative vagina, gastrointestinal tract, and skin care are also important factors for reducing post-operative infection, and bloating rates.

\section{DISCUSSION}

\subsection{Sufficient pre-operative preparation ensures less incidence probability of post-operative complications}

Surgical success includes patient providing effective psychological care which allows them to establish confidence in the treatment and cooperate with staff ${ }^{[3]}$. Pre-operative routine examinations ensure surgical safety, while the preoperative vagina, gastrointestinal tract, and skin care are also important factors for reducing post-operative infection, and bloating rates, pain site physical therapy, including patient-controlled analgesia (PCA), should occur to help patients recover normal gastrointestinal functions as early as possible. The surgery site should be observed for bleeding It is necessary to observe the bleeding conditions of the wound and vagina of the patients, reduce the accompanying pressure after the surgery and strengthen the working efficiency of surgical care to ensure the successful implementation of the surgery.

\subsection{Sufficient Post-operative Care Promotes the Quicker Recovery}

Post-operative observation can effectively reduce complications from bleeding. Catheter care, diet, and related activities can reduce the likelihood of infection, avoid pneumoperitoneum complications, bloating, vomiting, and other symptoms, to ensure a patient comfort. This will be conducive to postoperative nitrogen balances, accelerate healing, and speed rehabilitation ${ }^{[4]}$.

\subsection{Strengthen joint efforts and coordination between operation group staff and ward nurses}

Laparoscopic surgery success depends largely on coordinating surgery teams and ward nurses ${ }^{[5]}$. Preoperation preparation includes diet, vaginal and gastrointestinal cleaning, and a comprehensive mastery the dynamic conditions at various stages of surgery, nursing measures, and follow up to monitor and observe post-operative BP, R, and P changes. Catheterization is unnecessary. Physical therapy, ambulation, including trips to the toilet.

\section{CONCLUSION}

The advantages of laparoscopic panhysterectomies include smaller abdominal incisions, shorter hospital stays, improved bed turnover rates, lower infection rates, and quicker recoveries. It is superior to abdominal hysterectomies and more suitable clinically. Though warranting further study, perioperative care will benefit post-operative recoveries. 


\section{CONFLICT OF INTERESTS}

The authors declare no conflict of interests.

\section{FUNDINGS}

This research received no specific grant from funding agencies in the public, commercial, or not-for-profit sectors.

\section{REFERENCES}

1. Li X, Zhou Q. Perioperative nursing of hysterectomy with laparoscope. Modern J Integr
Trad Chinese Western Med. 2014; 23(26): 2124.

2. $\mathrm{Xu} \mathrm{Y}$. Perioperative Nursing of hysterectomy with laparoscope. Inner Mongol J Trad Chinese Med. 2014; 9 (27): 101-104.

3. Song L. Comparison of clinical effects between panhysterectomy and abdominal hysterectomy with laparoscope. Contemp Med. 2014; 20 (25): 91-94.

4. Duan L, Wang H, Liu Y. Analysis of complications with five cases with panhysterectomy injury with laparoscope. Chinese J Minim Inv Surg. 2015; 15(1): 79-81.

5. Hou S, Zhang X, Cao H. Clinical application of evidence-based care of patients in hysteromyomectomy with laparoscope. China Prac Med. 2015; 10(2): 131-133. 